\title{
Long-term outcomes of sleeve gastrectomy as a revisional procedure after failed gastric band: a multicenter cross-matched cohort study
}

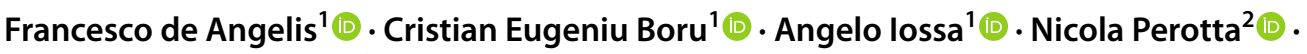 \\ Fabio Cesare Campanile ${ }^{3}$ (i) . Gianfranco Silecchia ${ }^{1}$ (I)
}

Received: 2 July 2021 / Accepted: 22 September 2021 / Published online: 3 October 2021

(c) The Author(s) 2021

\begin{abstract}
Laparoscopic adjustable gastric band (LAGB) is the bariatric procedure most likely subject to revisional surgery. Both laparoscopic sleeve gastrectomy (LSG) and Roux-en-Y gastric bypass (LRYGB) represent viable options, but the long-term results are still lacking. In 2014, we published the 2-year follow-up of our multicenter cohort of revisional LSG after failed LAGB. Evaluate the long-term follow-up (median 9.3 years) of the same cohort of patients. University and primary-care hospitals, Italy. We retrospectively examined a prospectively maintained database of the previously published multicenter cohort of 56 patients who underwent LSG after failed LAGB between 2008-2011. The control group included cross-matched non-revisional LSGs. The primary endpoint was weight loss, secondary endpoints co-morbidities, and the need for further bariatric surgery. The study group included 44 patients and the control group 56. We found \%EWL 53\% Vs. 67\% $(p=.021)$, \%EBMIL (54 Vs. 68\%, $p=.018$ ), \% TWL (26 Vs. 34\%, $p=.002$ ). We also found more severe GERD (gastroesophageal reflux disease) symptoms in the revisional than in the primary group (9.0 vs. $1.8 \%$ mild and 23.0 vs. $3.0 \%$ severe). Ten patients from the revisional group (22.7\%) vs. eight in the primary group (13\%) underwent further bariatric surgery (LRYGB). Our results showed less favorable weight loss in revisional than primary LSG after LABG, higher prevalence of GERD, and a more frequent need for further revisional surgery. Despite the study's limitations, the present data suggest that the long-term outcomes may offset the possible reduced short-term complication rate after revisional sleeve gastrectomy for a failed LABG.
\end{abstract}

Keywords Failed gastric banding · Revisional surgery · Sleeve gastrectomy · Weight regain · GERD · Gastric bypass

\section{Introduction}

Laparoscopic adjustable gastric band (LAGB) is the bariatric procedure most likely subject to revisional surgery [1]. Despite good short-term outcomes in weight loss and postoperative complications, it is associated with over $50 \%$

Francesco de Angelis and Cristian Eugeniu Boru contributed equally as first author.

Gianfranco Silecchia

gianfranco.silecchia@uniroma1.it

Francesco de Angelis

francescodeangelis7@gmail.com

Cristian Eugeniu Boru

drcrisb@gmail.com

Angelo Iossa

angelo.iossa@gmail.com

Nicola Perotta

perrott@tiscali.it long-term failure [1,2]. Although revisional surgery is considered safe and effective, there is still considerable heterogeneity in choosing the proper intervention after a failed primary procedure $[3,4]$. Both laparoscopic Roux-en-Y gastric bypass (LRYGB) and laparoscopic sleeve gastrectomy (LSG) represent viable options for failed LAGB; however, the long-term results for both revisional procedures are still lacking $[5,6]$. To our knowledge, there are no multicenter

Fabio Cesare Campanile campanile@surgical.net

1 Division of General Surgery \& Bariatric Center of Excellence IFSO-EC, Department of Medico-Surgical Sciences and Biotechnologies, University La Sapienza of Rome, Corso Della Repubblica, 78, 04100 Latina, Italy

2 Department of General Surgery, "San Pio da Pietralcina" Hospital, Villa D’Agri, Italy

3 Division of General Surgery, Andosilla Hospital, Civita Castellana, Italy 
cohort studies with a long-term follow-up after revisional LSG following LAGB [7].

In 2014, we published the 2-year follow-up of our multicenter cohort of revisional LSG in a "2-step" approach after failed LAGB. In 56 patients, we reported excess weight loss (\%EWL) of $78.5 \%$ without major complications. The results were not statistically different from those obtained in our primary (not revisional) sleeve gastrectomy series [8].

The present, multicenter, retrospective cohort study aimed to update the long-term results of LAGB patients converted to LSG, providing a 9-year follow-up of that series.

\section{Methods}

We retrospectively examined a prospectively maintained database of the previously published cohort (retrospective cohort study) [8]. Three Italian bariatric centers were involved in the study, which was approved by the Institutional Review Board (SCDSBMC/27.05.2020 University La Sapienza of Rome) and the Local Committees. The same 56 patients of the published short-term study were included in the present study group; they underwent LSG after failed LAGB between 2008 and 2011 ("revisional group"). The results were compared to patients selected from the original study's not-revisional control group, cross-matched for age, sex, weight, and BMI ("primary group").

Revisional surgery had been indicated for inadequate EWL $(<30 \%)$, weight regain, and LAGB-related complications. The choice for LSG as a revisional procedure was based on the multidisciplinary re-evaluation of the alimentary and lifestyle behavior and absence of gastroesophageal reflux disease (GERD). The preoperative workup included upper gastro-intestinal contrast studies and endoscopy. No hiatal hernia was found at LSG.

The surgical technique for band removal, sleeve gastrectomy, and perioperative management was similar in all centers. The exclusion criteria, demographics, and the study flow-chart are reported in Table 1 and Fig. 1. All patients were evaluated yearly by a multidisciplinary team. The gastroesophageal reflux disease GERD Health-Related Quality of Life Questionnaire (GERD-HRQL) was administered yearly starting from 2016, to all operated patients, during the scheduled outpatient evaluation [9]. GERD symptoms were defined as absent when patients reported a GERD-HRQL score of 0 , mild from 1 to 15 , moderate from 16 to 30 , and severe from 31 to 50 .

Insufficient weight loss was defined as $\mathrm{EWL}<50 \%$ one year postoperatively; weight regain as an increase of more than $10 \mathrm{~kg}$ from nadir (lowest weight obtained) [10].

All patients that underwent further revisional surgery had a multidisciplinary re-evaluation (nutritional and psychological counseling included), upper GI endoscopy, contrast
Table 1 Demographics of revisional LSG after failed band vs. crossmatched primary LSG

\begin{tabular}{llll}
\hline Characteristics & Revisional group & Primary group & $p$ value \\
\hline $\begin{array}{l}\text { Number } \\
\text { Gender }\end{array}$ & 44 & 56 & \\
$\quad$ Male & $10(22.7 \%)$ & $8(17.7 \%)$ & \\
Female & $34(77.3 \%)$ & $48(82.3 \%)$ & \\
Age: median (range) & $46(21-59)$ & $42(20-59)$ & $p=.600$ \\
Weight in Kg: median & $114(90-175)$ & $124(98-213)$ & $p=.056$ \\
$\quad$ range) & & & \\
$\begin{array}{l}\left.\text { BMI (Kg/m }{ }^{2}\right): \text { median } \\
\text { Comorbidities }\end{array}$ & $42.51(32.6-56.5)$ & $44.41(37-54)$ & $p=.161$ \\
$\quad$ & & & \\
Diabetes Type II & $7(15.9 \%)$ & $11(19.64 \%)$ & $p=.632$ \\
Hypertension & $20(45.45 \%)$ & $24(42.85 \%)$ & $p=.661$ \\
\hline
\end{tabular}

$\mathrm{X}$-ray. Esophageal manometry and $\mathrm{pH}$-metry were obtained in selected cases, or a CT scan with $3 \mathrm{D}$ reconstruction to rule out a hiatal defect or intrathoracic sleeve migration [11]. Non-responder GERD patients were referred for conversion to Roux-en-Y Gastric Bypass (RYGB), with posterior cruroplasty indicated. If the hiatal defect was associated with weak pillars, a biosynthetic, absorbable scaffold prosthesis was added to the cruroplasty [12].

The primary endpoint was weight loss after revisional LSG in terms of percent excess weight loss (\%EWL), percent of total weight loss (\%TWL), percent excess BMI loss (\%EBMIL) [13]. Secondary endpoints were resolution, relapse, or de novo occurrence of major co-morbidities and the need for further bariatric procedures after failed revisional surgery for any reason.

The statistical analysis was performed using Statistica10 (TIBCO Software Inc., CA, USA). Normally distributed quantitative variables were expressed as mean with standard deviation, median with the min-max range in skewed data, qualitative variables as a percentage. The Student $t$-test and Chi-Square test were used to compare continuous and categorical data. $p$ values $<0.05$ (two tailed) were considered significant.

\section{Results}

After a median follow-up of 9.3 years (range 8.0-12.8 years), 44 out of the initial 56 patients included in the first report were available for the study's completion (10 males and 34 females). Their median initial BMI was $42.51 \mathrm{~kg} / \mathrm{m}^{2}$ (range 32.6-56.5). No patient died during the follow-up. Twelve patients were unavailable for the study (one for a severe disability, two for cognitive impairment due to senile dementia and nine for refusal to participate in the scheduled controls). 
Fig. 1 Study's flow-chart of revisional LSG after failed gastric banding vs. primary cross-matched LSG

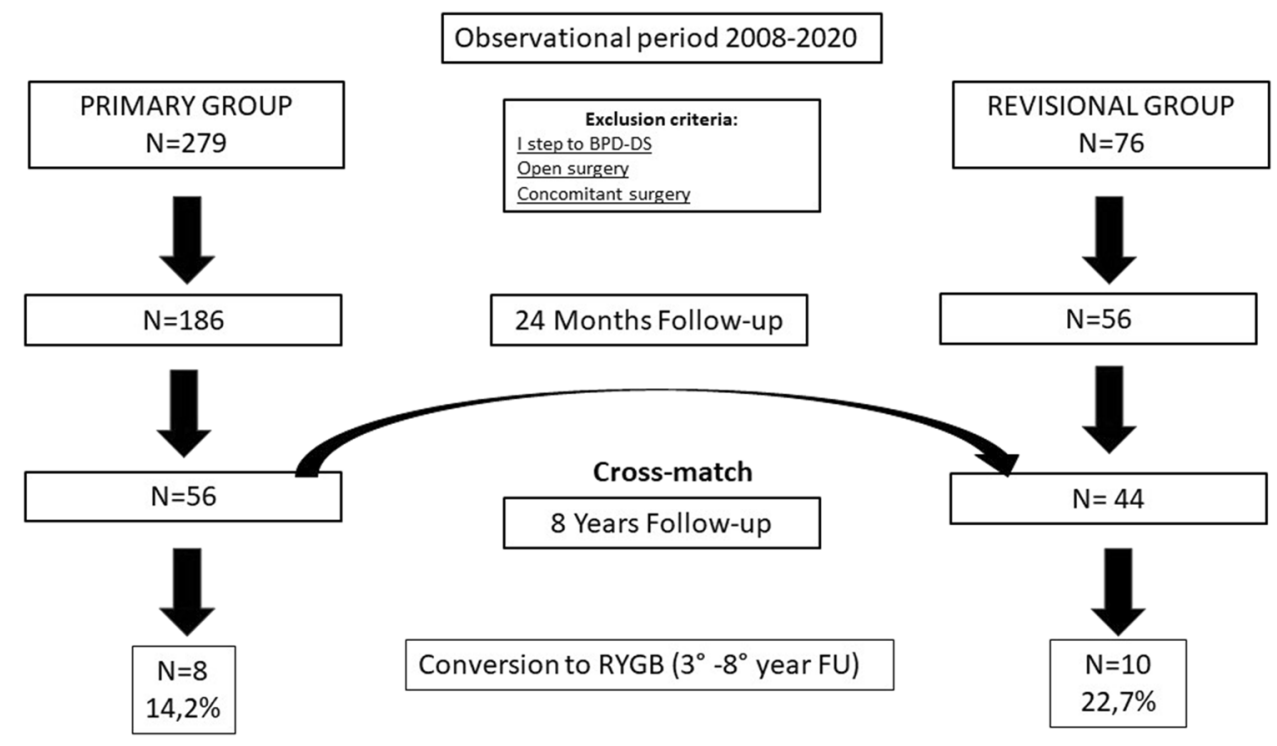

Table 2 EWL\%, TWL\% and EBMI\% after 8 years follow-up of revisional LSG after failed band vs. cross-matched primary LSG

\begin{tabular}{llll}
\hline & RG 8 year & PG 8 year & $p$ value \\
\hline EWL \% & $53 \pm 26$ & $67 \pm 27$ & 0.021 \\
TWL \% & $26 \pm 12$ & $34 \pm 12$ & 0.002 \\
EBMIL \% & $54 \pm 25$ & $68 \pm 27$ & 0.018 \\
\hline
\end{tabular}

Data are expressed as mean $\pm \mathrm{SD}$

$E W L$ Excess weight loss, $T W L$ Total weight loss, EBMIL Excess BMI loss, $R G$ Revisional group, $P G$ Primary group

The matched control group included 56 not-revisional patients ( 8 males and 48 females) with a median BMI of $44.41 \mathrm{~kg} / \mathrm{m}^{2}$ (range 37-54). All of them were available for follow-up.

The \%EWL, \%EBMIL and \%TWL were significantly different and indicated lower weight loss after revisional surgery. The data are shown in Table 2.

The GERD-HRQL questionnaires' analysis reported that in the revisional group, 30 patients $(68 \%)$ were asymptomatic, 4 (9\%) mildly and $10(23 \%)$ severely symptomatic. In the primary group, there were $53(95 \%)$ asymptomatic, $1(1.8 \%)$ mildly and $2(3 \%)$ severely symptomatic patients $(p=0.01)$. In the revisional group, $34 \%$ of patients used proton pump inhibitors (PPIs) to treat GERD (either selfmedication or prescribed by the general practitioner), vs. only $8(12 \%)$ of primary group.

Ten patients from the revisional group (22.7\%) and eight from the primary group (14\%) underwent further laparoscopic RYGB. The indication to re-revision was insufficient weight loss or weight regain ( 2 vs. 5 patients) and severe GERD associated with a grade B esophagitis or higher (8 vs.
3 patients) [13, 14]. A concomitant type I hiatal hernia was detected in 5 revisional patients and none of the controls.

At the end of the follow-up, hypertension was present in $18.1 \%$ of the revisional vs. $5.3 \%$ of the primary group (preoperative rate: 45.4 vs. $42.8 \%$ ), and type II diabetes in $2.3 \%$ Vs. nil (preoperative rate: 15.9 vs. $19.6 \%$ ).

\section{Discussion}

The long-term follow-up (median: 9.3 years) of our series of revisional LSG following LAGB demonstrated lower weight loss than in the primary LSG control group and a higher prevalence of severe GERD. GERD was also the main reason to convert a revisional sleeve gastrectomy to RYGB (8 out of 10 in the revisional group vs. 3 out of 8 in the primary group).

LSG and RYGB have been proposed as effective revisional procedures after failed LAGB. Both show good shortterm outcomes and acceptable postoperative complication rates [1-6]. It is controversial which of the two procedures is more effective on long-term ( $>5$ years) weight loss, comorbidities control, and complications [3, 6].

Angrisani et al. found no statistically significant difference in percent excess weight loss in patients converted to LSG or RYGB [15]. Other studies showed less weight loss with conversion to LSG when compared with RYGB [16] and less weight loss than primary LSG, as in our present experience.

Recent evidence supports that single-step and two steps approaches are both safe [16, 17]. In both approaches, conversion to LSG might determine fewer short-term post-op complications than RYGB [4, 17]. 
In 2014, we evaluated the short-term follow-up of a cohort that underwent revisional LSG after failed LAGB in a "2-step" approach; the results demonstrated a good weight loss at 24 months, associated with low major complications rate.

The longer follow-up of the same series, included in the present work, provides evidence that de novo, severe GERD manifests as a mid-term complication and often requires further revisional surgery. Similar findings were shown in nonrevisional sleeve gastrectomy [18, 19].

Our findings suggest that when conversion to LSG rather than RYGB is selected, the burden of long-term GERD may balance the advantage of a lower short-term complication rate.

The finding of type I hiatal hernia in $50 \%$ of our revisional patients who underwent conversion to RYGB deserves attention. We did not find any hiatal hernia in patients who had RYGB after a failed primary LSG. The significantly higher prevalence in the study group can hardly be related to a lack of hiatus dissection at the previous LSG: even if an occasional hiatal hernia could have been missed, we systematically explored the gastroesophageal junction at the conversion surgery, as previously described. One hypothesis is that the extensive dissection, due to the scarring of the previous gastric band site, could have weakened the hiatal area and compromised some of the natural contention methods.

In the future, the difference between the two groups in terms of "de novo" GERD incidence, despite the systematic hiatus exploration, may influence the revisional operation's choice. The patients who required LSG's revisions to RYGB did not show any postoperative complications in both groups, but other series' rate is up to $25 \%$ [19-22].

The present study includes all the known limitations of the small-size retrospective design. The case-matching could also have missed relevant confounding factors. In addition, the high $(21.4 \%)$ dropout rate from the previous study group could have introduced a selection bias and limited the ability to control confounding control by the matching design. Finally, as per an intention-to-treat design, we administered the GERD-HRQL questionnaire at the end of the study period, regardless of the patient having had an additional revising procedure (RYGB) or not. This aspect affected the results about GERD symptoms.

However, this is the first long-term multicentric retrospective study on revisional 2-stage LSGs after failed LAGB. Moreover the prospectively maintained database available for the entire follow-up, adds strengthens the study's design.

\section{Conclusion}

Our results confirmed less favorable weight loss in revisional than primary LSG after LABG. In the revisional group, the incidence of GERD and the need for further revisional surgery are higher. Despite the study's limitations, the present data suggest that the long-term outcomes may offset the possible reduced short-term complication rate after revisional sleeve gastrectomy. Short- and long-term consequences should be included in an exhaustive discussion with the patient candidate to the revision.

Funding Open access funding provided by Università degli Studi di Roma La Sapienza within the CRUI-CARE Agreement. No funding was provided for this study.

Availability of data and materials On request.

\section{Declarations}

Conflict of interests All authors have no conflict of interest.

Consent for publication All authors give their consent for publication.

Ethical approval All procedures performed in the study were in accordance with ethical standards of the institutional and/or national research committee and with the 1964 Helsinki declaration and its later amendments or comparable ethical standards.

Informed consent For this type of study formal consent is not required by local regulations.

Open Access This article is licensed under a Creative Commons Attribution 4.0 International License, which permits use, sharing, adaptation, distribution and reproduction in any medium or format, as long as you give appropriate credit to the original author(s) and the source, provide a link to the Creative Commons licence, and indicate if changes were made. The images or other third party material in this article are included in the article's Creative Commons licence, unless indicated otherwise in a credit line to the material. If material is not included in the article's Creative Commons licence and your intended use is not permitted by statutory regulation or exceeds the permitted use, you will need to obtain permission directly from the copyright holder. To view a copy of this licence, visit http://creativecommons.org/licenses/by/4.0/.

\section{References}

1. Tsai C, Zehetner J, Beel J, Steffen R (2019) Long-term outcomes and frequency of reoperative bariatric surgery beyond 15 years after gastric banding: a high band failure rate with safe revisions. Surg Obes Relat Dis 15(6):900-907. https://doi.org/10.1016/j. soard.2019.03.017

2. Di Lorenzo N, Antoniou SA, Batterham RL et al (2020) Clinical practice guidelines of the European Association for Endoscopic Surgery (EAES) on bariatric surgery: update 2020 endorsed by IFSO-EC, EASO and ESPCOP. Surg Endosc 34(6):2332-2358. https://doi.org/10.1007/s00464-020-07555-y

3. Mahawar KK, Graham Y, Carr WR et al (2015) Revisional Roux-en-Y gastric bypass and sleeve gastrectomy: a systematic review of comparative outcomes with respective primary procedures. Obes Surg 25(7):1271-1280. https://doi.org/10.1007/ s11695-015-1670-2

4. Carandina S, Maldonado PS, Tabbara M et al (2014) Two-step conversion surgery after failed laparoscopic adjustable gastric banding. Comparison between laparoscopic Roux-en-Y gastric 
bypass and laparoscopic gastric sleeve. Surg Obes Relat Dis 10(6):1085-1091. https://doi.org/10.1016/j.soard.2014.03.017

5. Sharples AJ, Charalampakis V, Daskalakis M, Tahrani AA, Singhal R (2017) Systematic review and meta-analysis of outcomes after revisional bariatric surgery following a failed adjustable gastric band. Obes Surg 27(10):2522-2536. https://doi.org/10.1007/ s11695-017-2677-7

6. Zhou R, Poirier J, Torquati A, Omotosho P (2019) Short-term outcomes of conversion of failed gastric banding to laparoscopic sleeve gastrectomy or Roux-En-Y gastric bypass: a metaanalysis. Obes Surg 29(2):420-425. https://doi.org/10.1007/ s11695-018-3538-8

7. Carandina S, Genser L, Bossi M, Polliand C, Tabbara M, Barrat C (2017) Laparoscopic sleeve gastrectomy after failed gastric banding: is it really effective? Six years of follow-up. Surg Obes Relat Dis 13(7):1165-1173. https://doi.org/10.1016/j.soard.2017.02.008

8. Silecchia G, Rizzello M, De Angelis F et al (2014) Laparoscopic sleeve gastrectomy as a revisional procedure for failed laparoscopic gastric banding with a "2-step approach": a multicenter study. Surg Obes Relat Dis 10(4):626-631. https://doi.org/10. 1016/j.soard.2013.10.017

9. Balla A, Leone G, Ribichini E et al (2020) Gastroesophageal reflux disease-health-related quality of life questionnaire: prospective development and validation in Italian. Eur J Gastroenterol Hepatol. https://doi.org/10.1097/MEG.0000000000001914 (Epub ahead of print)

10. Lauti M, Kularatna M, Hill AG, MacCormick (2016) Weight regain following sleeve gastrectomy-a systematic review. Obes Surg 26(6):1326-1334

11. Rengo M, Bellini D, Iorio O et al (2013) Role of preoperative imaging with multidetector computed tomography in the management of patients with gastroesophageal reflux disease symptoms after laparoscopic sleeve gastrectomy. Obes Surg 23(12):19811986. https://doi.org/10.1007/s11695-013-1003-2

12. Boru CE, Greco F, Giustacchini P, Raffaelli M, Silecchia G (2018) Short-term outcomes of sleeve gastrectomy conversion to R-Y gastric bypass: multi-center retrospective study. Langenbecks Arch Surg 403(4):473-479. https://doi.org/10.1007/s00423-018-1675-0

13. Brethauer SA, Kim J, El Chaar M, ASMBS Clinical Issues Committee et al (2015) Standardized outcomes reporting in metabolic and bariatric surgery. Surg Obes Relat Dis 11(3):489-506. https:// doi.org/10.1016/j.soard.2015.02.003

14. Lundell LR, Dent J, Bennett JR et al (1999) Endoscopic assessment of oesophagitis: clinical and functional correlates and further validation of the Los Angeles classification. Gut 45(2):172-180
15. Angrisani L, Vitiello A, Santonicola A, Hasani A, De Luca M, Iovino P (2017) Roux-en-Y gastric bypass versus sleeve gastrectomy as revisional procedures after adjustable gastric band: 5-year outcomes. Obes Surg 27(6):1430-1437. https://doi.org/10.1007/ s11695-016-2502-8

16. Creange $C$, Jenkins $M$, Pergamo M, Fielding G, Ren-Fielding C, Schwack B (2018) Gastric band conversion to Roux-en-Y gastric bypass shows greater weight loss than conversion to sleeve gastrectomy: 5-year outcomes. Surg Obes Relat Dis 14:1531-1536. https://doi.org/10.1016/j.soard.2018.06.002

17. Janik M, Ibikunle C, Khan A, Aryaie AH (2021) Safety of single stage revision laparoscopic sleeve gastrectomy compared to laparoscopic Roux-Y gastric bypass after failed gastric banding. Obes Surg 31(2):588-596. https://doi.org/10.1007/s11695-020-04975-6

18. Dang JT, Switzer NJ, Wu J et al (2016) Gastric band removal in revisional bariatric surgery, one-step versus two-step: a systematic review and meta-analysis. Obes Surg 26(4):866-873. https://doi. org/10.1007/s11695-016-2082-7

19. Perry Z, Romano-Zelekha O, Sakran N et al (2021) Laparoscopic sleeve gastrectomy following failed laparoscopic adjustable gastric banding-a comparison between one- and two-stage procedures, an israeli national database study. Obes Surg. https://doi. org/10.1007/s11695-021-05312-1

20. Felsenreich DM, Kefurt R, Schermann M et al (2017) Reflux, sleeve dilation, and Barrett's esophagus after laparoscopic sleeve gastrectomy: long-term follow-up. Obes Surg 27(12):3092-3101. https://doi.org/10.1007/s11695-017-2748-9

21. DuPree CE, Blair K, Steele SR, Martin MJ (2014) Laparoscopic sleeve gastrectomy in patients with preexisting gastroesophageal reflux disease: a national analysis. JAMA Surg 149:328-334

22. Amiki M, Seki Y, Kasama K et al (2020) Revisional bariatric surgery for insufficient weight loss and gastroesophageal reflux disease: our 12-year experience. Obes Surg 30(5):1671-1678. https://doi.org/10.1007/s11695-019-04374-6

Publisher's Note Springer Nature remains neutral with regard to jurisdictional claims in published maps and institutional affiliations. 